\title{
Endogenous Growth: A Knife Edge or the Razor's Edge?*
}

\section{Carl-Johan Dalgaard}

University of Copenhagen, DK-1455 Copenhagen, Denmark

carl.johan.dalgaard@econ.ku.dk

\section{Claus Thustrup Kreiner}

University of Copenhagen, DK-1455 Copenhagen, Denmark

claus.thustrup.kreiner@econ.ku.dk

\begin{abstract}
According to much of the recent growth literature, the dramatic worldwide decline in fertility currently taking place should ultimately lead to global economic stagnation. This pessimistic prediction is not shared by the original innovation-based growth literature. In recent years, however, this strand of the literature has been criticized for resting on implausible knife-edge assumptions and for its inconsistency with available evidence. In this paper, we argue that this conclusion is unwarranted.
\end{abstract}

Keywords: Endogenous growth theory

JEL classification: 040

\section{Introduction}

Recent population estimates by the United Nations (1998) emphasize the need for establishing whether long-run economic growth is feasible in the absence of population growth. On all continents of the world and, in particular, in regions where the bulk of global $\mathrm{R} \& \mathrm{D}$ efforts are taking place (i.e., the US, Japan and Western Europe), fertility is rapidly declining. In fact, even if immigration is taken into account, the total population in the G7 area in 2050 is expected to be roughly the same as in 1998. These demographic conditions can be expected to entail the ultimate demise of global growth in living standards if an expanding population is deemed necessary for perpetual (exponential) growth. Indeed, according to one strand of the literature on innovation-based growth, perpetual increases in

\footnotetext{
*We would like to thank Oded Galor, Christian Groth and Jacob Weisdorf for helpful comments and suggestions. We are also grateful for improvements suggested by two anonymous referees. This research was carried out at EPRU (Economic Policy Research Unit), whose activities are financed through a grant from the Danish National Research Foundation.
} 
living standards can only be achieved through continual population growth; see e.g. Jones (1995b) and Segerstrom (1998). In contrast to these "semiendogenous" growth models, the original innovation-based endogenous growth literature delivers less gloomy outlooks by claiming that growth in income per capita is possible without increases in population size; cf. Romer (1990) Grossman and Helpman (1991) and Aghion and Howitt (1992). ${ }^{1}$ But which theory should be relied on when assessing the prospects for future income growth?

Owing to two distinct arguments against endogenous growth theory, the received perception seems to be that semi-endogenous growth theory is superior. The main point of this paper is to demonstrate that these two arguments rely on specific functional forms, which are chosen solely for analytical convenience.

The first argument is that endogenous growth constitutes a "knife-edge case" since it requires exactly constant returns to producible (and essential) factors of production. This feature seems quite unrealistic in the sense that the standard replication argument cannot be applied to the production of factors such as scientific knowledge and human capital. Moreover, it is well known that small deviations from constant returns dramatically alter the nature of the growth equilibrium. If slightly increasing returns to producible factors are allowed, growth will "explode"; if slightly decreasing returns to producible factors are imposed, growth will come to a halt in the absence of population growth. Hence, from this perspective, endogenous growth theory seems to be a rather special case. Indeed, according to Solow (1994, p. 51):

"The knife-edge character of the constant-returns model cannot be evaded... The conclusion has to be that this version of the endogenousgrowth model is very un-robust. It can not survive without exactly constant returns to capital. But you would have to believe in the tooth fairy to expect that kind of luck."

While Solow's criticism was directed primarily towards the first wave of endogenous growth models, i.e., the AK model as in Frankel (1962) and Romer (1986), others such as Jones (1999) and $\operatorname{Li}(2000,2002)$ have recently argued that the innovation-based endogenous growth theory initiated by

\footnotetext{
${ }^{1}$ The term "semi-endogenous growth" was originally coined by Jones (1995). A model is said to exhibit semi-endogenous growth if the growth rate in per capita income is determined by an (some) exogenous - non-technological-growth rate(s). However, for all practical purposes, this implies that permanent growth hinges entirely on population growth since it is hard to think of any non-producible factor of production, other than the number of individuals in the population, that can continue to grow.
}

(C) The editors of the Scandinavian Journal of Economics 2003. 
Romer (1990), Grossman and Helpman (1991), and Aghion and Howitt (1992) rests on a similar knife-edge assumption.

In this paper we argue that the theory of innovation-based endogenous growth is more general than it appears. The argument is simple. In general, the knowledge production function may exhibit decreasing returns, increasing returns, or perhaps alternate over time between decreasing and increasing returns. The crucial condition for endogenous growth is that the marginal product of knowledge in producing new ideas converges towards some positive constant in the long run.

From a technical perspective, this argument builds on Jones and Manuelli (1990) who demonstrated that perpetual growth may arise in the standard neoclassical growth model when the marginal product of capital is bounded from below by a positive constant, thus violating one of the Inada conditions. ${ }^{2}$ In contrast, we focus on the marginal productivity of knowledge in creating new ideas. This difference in scope is more than semantic. Whereas the Inada condition - which states that the marginal product of physical capital tends to zero as the capital stock tends to infinity (given other factors of production such as labor or land) - might seem reasonable, it seems much less intuitive when imposed on the marginal productivity of the stock of knowledge in producing new ideas. The central question is this: why would a new piece of information be completely unproductive in producing new ideas even if there did in fact exist infinitely many other pieces of information? ${ }^{3}$

The second argument against the theory of endogenous growth is that it is inconsistent with available evidence. For example, the models predict that economic policy, and incentives in general, can affect the growth rate in the long run. But as pointed out by Jones (1995a), this proposition seems inconsistent with the empirical evidence; time-series data for several OECD countries covering the last few decades show that the growth rate in income per capita has remained remarkably stable even though tax structures have changed substantially. ${ }^{4}$ In addition, endogenous growth models have been criticized for being at variance with observed convergence patterns; see e.g. Eicher and Turnovsky (1999b). By way of contrast we argue that endogenous growth theory may be consistent with these observations.

\footnotetext{
${ }^{2}$ This point was made early on by Pitchford (1960).

${ }^{3}$ In addition, assuming that the marginal productivity of knowledge in producing new ideas is bounded away from zero asymptotically, does not lead to the unwelcome implication that the capital share of total income tends to one in the long run, as is the case in Jones and Manuelli (1990).

${ }^{4}$ Jones also notes that the number of scientists has increased rapidly during this period. This is inconsistent with endogenous growth models, which feature a scale effect on the growth rate from the number of scientists. By now, "however, several papers have shown that this prediction may be avoided in endogenous growth theory; cf. Young (1998), Howitt (1999) and Dalgaard and Kreiner (2001).
} 


\section{On the Generality of Perpetual Growth in the Absence of Population Growth}

We consider a simple reduced-form framework. Final goods, $Y$, are produced according to

$$
Y=F\left(A, L ; \alpha_{Y}\right), \quad 0 \leqslant \alpha_{Y} \leqslant \bar{\alpha}_{Y}
$$

where $F(\cdot)$ is a standard neoclassical production function, $A$ is the stock of (scientific) knowledge, and $L$ is labor input. Since our focus is on growth driven by $\mathrm{R} \& \mathrm{D}$, we exclude other reproducible factors of production - such as physical capital and human capital - although such inputs could be added without altering the arguments that follow in any essential way. ${ }^{5}$ Similarily, other non-producible inputs aside from labor could also be added. However, this would contribute nothing, since labor is the only non-producible input which can continue to grow.

For given stocks of $A$ and $L$, production may change due to the shift parameter $\alpha_{Y}$. For example, $\alpha_{Y}$ could represent the fraction of labor used in final goods production, or it may more generally represent economic institutions that are important for the effectiveness of inputs.

The $R \& D$ equation is given by

$$
\dot{A}=H\left(A, L ; \alpha_{A}\right), \quad 0 \leqslant \alpha_{A} \leqslant \bar{\alpha}_{A},
$$

where $H>0, H_{1} \gtreqless 0, H_{2} \geqslant 0$, and $\alpha_{A}$ is a shift parameter. ${ }^{6}$ Note that the two shift parameters may be constrained by each other. If, for instance, the two parameters denote the fraction of labor input used in the two types of production, respectively, then the two parameters are constrained according to $\alpha_{Y}+\alpha_{A}=1$. However, our results are independent of whether such constraints exist or not.

The literature on innovation-based growth usually adopts special functional forms. For instance, it is common to assume $F\left(A, L ; \alpha_{Y}\right)=A^{\gamma}\left(\alpha_{Y} L\right)$ and $H\left(A, L ; \alpha_{A}\right)=\delta A^{\epsilon}\left(\alpha_{A} L\right)^{\eta}$, where $\alpha_{Y}+\alpha_{A}=1$. The parameters $\gamma, \delta$ and $\eta$

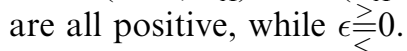

Endogenous growth arises only when $\epsilon=1$, in which case the growth rate in income, $g_{Y} \equiv \dot{Y} / Y$, becomes

$$
g_{Y}=\gamma \delta\left(\alpha_{A} L\right)^{\eta}
$$

\footnotetext{
${ }^{5}$ In fact, $A$ could be regarded as a composite input of different producible factors of production including scientific knowledge.

${ }^{6}$ The case $H_{1}<0$ corresponds to the "fishing-out" scenario; see e.g. Segerstrom (1998).

(C) The editors of the Scandinavian Journal of Economics 2003.
} 
where $L$ is assumed fixed. Accordingly, perpetual growth does not hinge on an expanding population. However, it may now be argued that this theory of endogenous growth is a rather special case, i.e., a knife-edge case, since it requires exactly constant returns to knowledge in producing new ideas. Again, this feature might seem unrealistic since the standard replication argument cannot be applied to the production of knowledge. Moreover, if $\epsilon$ is just slightly above one, growth explodes. On the other hand, if $\epsilon$ is just slightly below one, the nature of the growth process changes in a fundamental way. In particular, the steady-state growth rate equals

$$
g_{Y}=\left(\frac{\gamma \eta}{1-\varepsilon}+1\right) n
$$

where $n$ is the population growth rate, which is assumed exogenous and constant. Thus, population growth is required in order to keep economic growth in momentum. This so-called semi-endogenous growth theory arises for all values of $\epsilon$ below one and has therefore been argued to be more plausible than the theory of endogenous growth. However, this argument is misleading.

Going back to equation (1) and differentiating with respect to time, we have

$$
g_{Y}=\frac{F_{1} A}{Y} \frac{\dot{A}}{A}+\frac{F_{2} L}{Y} \frac{\dot{L}}{L}
$$

In order to sustain innovation-based perpetual growth, it is nessesary to assume that $F_{1} A / Y>0$. Except for this assumption, the production function for final goods is not crucial. From equation (2), we obtain the rate of growth in knowledge

$$
g_{A}=\frac{H\left(A, L ; \alpha_{A}\right)}{A} \equiv h\left(A, L ; \alpha_{A}\right) .
$$

With the specific functional forms considered above, $h\left(A, L ; \alpha_{A}\right)$ is independent of the first argument in the case of endogenous growth and decreasing in the case of semi-endogenous growth. Thus, this feature of the $h(\cdot)$ function seems crucial in determining the type of growth. But this is not so. If we exclude the possibility of explosive growth, then the growth rate of $A$ will, for a given $L$, converge towards some constant, i.e.,

$$
\lim _{A \rightarrow \infty} h\left(A, L ; \alpha_{A}\right)=\tilde{h}\left(L ; \alpha_{A}\right)
$$

where $\tilde{h}$ is a constant that may depend on the parameter $\alpha_{A}$. This yields 
Proposition. Perpetual growth arises in the absence of population growth if $\tilde{h}\left(L ; \alpha_{A}\right)>0$. Perpetual growth requires population growth if $\tilde{h}\left(L ; \alpha_{A}\right)=0$.

Hence, endogenous growth is compatible with decreasing, constant and increasing returns to knowledge in creating new ideas. The only restriction is that $h\left(A, L ; \alpha_{A}\right)$ asymptotically tends to a strictly positive constant. ${ }^{7}$ This is illustrated in Figure 1 where curve 1 corresponds to exactly constant returns, curve 2 to decreasing returns and curve 3 to increasing returns, whereas curve 4 shows a case where the returns to scale change as a function of the stock of knowledge. All of these curves converge towards $\bar{h}$ and therefore feature an endogenous stock of knowledge. All of these curves converge toward $\bar{h}$ and therefore endogenous growth. Semi-endogenous

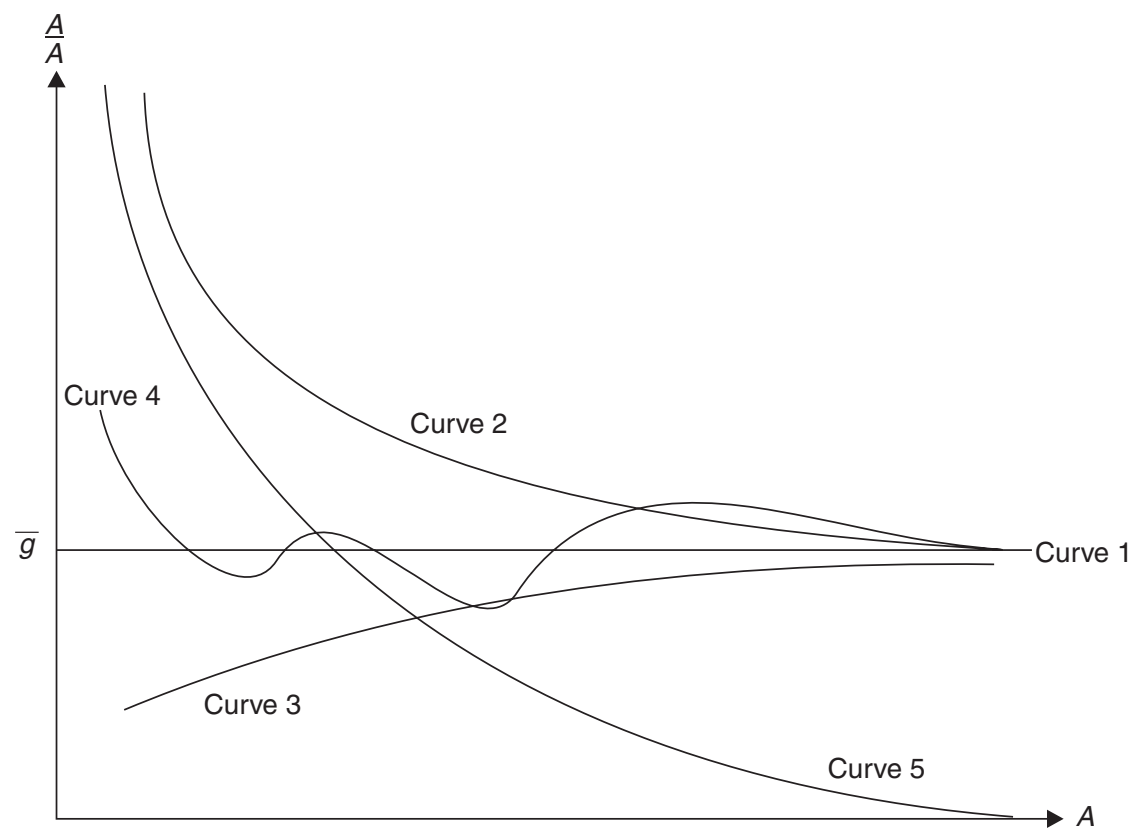

Fig. 1. Knowledge growth as a function of the stock of knowledge

\footnotetext{
${ }^{7}$ Weitzman (1998) provides some microfoundations for the R\&D process that point toward an ever expanding number of ideas, i.e., for the condition $\lim _{A \rightarrow \infty} H_{1}>0$. The argument builds on the presumption that scientific progress often takes on the shape of combining existing pieces of knowledge in new ways. On this basis the number of ideas will tend to increase over time by combinatorial expansion. Eventually, however, growth will (or rather might) tend toward a constant due to mankind's limited capacity to process an ever increasing abundance of new ideas.
} 
growth. Semi-endogenous growth, on the other hand, occurs only when $h\left(A, L ; \alpha_{A}\right)$ tends asymptotically to zero as illustrated by curve 5 in Figure $1 .{ }^{8}$

Note that in the case of endogenous growth $\lim _{A \rightarrow \infty} h\left(A, L ; \alpha_{A}\right)=$ $\lim _{A \rightarrow \infty} H_{1}>0$, where the equality follows from l'Hôpital's rule. Hence, what endogenous growth requires is that an incremental increase in the stock of knowledge, when this stock is already "large", leads to some new ideas. Semi-endogenous growth theory, on the other hand, arises when an incremental increase in the stock of knowledge asymptotically leads to no new ideas, i.e., $\lim _{A \rightarrow \infty} H_{1} \leqslant 0$.

A simple example that illustrates some of the issues involved can be constructed using a CES function for knowledge production, i.e.,

$$
H\left(A, L ; \alpha_{A}\right)=\left[(\phi A)^{(\sigma-1) / \sigma}+\left((1-\phi) \alpha_{A} L\right)^{(\sigma-1) / \sigma}\right]^{\sigma /(\sigma-1)}, \quad \dot{L} / L=n,
$$

where the parameters fulfill $0 \leqslant n<\phi<1, \sigma>0$ and $\sigma \neq 1$. Owing to strict concavity of the specified $H$ function, the growth rate of knowledge is always a decreasing function of the stock of knowledge. Thus, the example only illustrates cases like "curve 2" or "curve 5" in Figure 1. Whether the knowledge function is described by curve 2 or curve 5 depends on whether the elasticity of substitution, $\sigma$, is above one or below one. Accordingly, the evolution of the economy depends crucially on whether $\sigma>1$ or $\sigma<1$. From equation (6), we obtain the following expression governing the growth rate of knowledge:

$$
\dot{g}_{A}=\left[1-\left(\phi / g_{A}\right)^{(\sigma-1) / \sigma}\right]\left[n-g_{A}\right] g_{A} .
$$

This equation gives rise to the two phase diagrams in Figures $2 a$ and $2 b$. Technically speaking, there are three steady states corresponding to $g_{A}=0$, $g_{A}=n$ and $g_{A}=\phi$. However, in the case where $\sigma>1$, the average product of knowledge, or equivalently $g_{A}$, is bounded from below by $\phi$, implying that the economy can never venture into the shaded area. Thus, the growth rate of knowledge will gradually decline and ultimately approach $\phi$. Accordingly, the long-run growth rate depends neither on the size nor the growth rate of population when the elasticity of substitution is above one. On the other hand, if the elasticity of substitution is below one, the average product of knowledge lies in the range between zero and $\phi$, and the growth rate of knowledge will therefore converge towards $n$. Hence, in this case, exponential growth in knowledge will eventually cease unless the population continues to expand.

In the literature, a strictly concave R\&D production function such as equation (6) normally gives rise to semi-endogenous growth. However, this

${ }^{8}$ Of course, curve 5 need not be monotonically decreasing toward zero. 
is due to the focus on Cobb-Douglas functions, i.e., on an elasticity of substitution exactly equal to one. ${ }^{9}$ Unfortunately, our knowledge of the $R \& D$ production function is limited. Specifically, we do not know whether the elasticity of substitution is above or below one. ${ }^{10}$ Certainly, there is no particular reason to believe in exactly unity. Thus, to conclude, it is impossible to say which theory, endogenous growth or semi-endogenous growth, is the more realistic, even when we confine ourselves to a strictly concave $\mathrm{R} \& \mathrm{D}$ production function.

\section{Empirical Issues}

A second line of critique raised against endogenous growth theory is empirically founded. Specifically, it has been argued that endogenous growth theory involves unrealistic speeds of convergence and is inconsistent with evidence on the effects of policy on growth. We revisit these two issues in turn.

The speed of convergence is defined as $\Phi=\left[\dot{g}_{a}(t)-\dot{g}_{a}^{*}(t)\right] /\left[g(t)-g^{*}(t)\right]$, that is the rate at which the growth rate of knowledge approaches its longrun value. ${ }^{11}$ It follows immediately that

$$
\lim _{g_{a} \rightarrow g^{*}} \Phi=\left.\frac{\partial \dot{g}_{a}}{\partial g_{a}}\right|_{g_{a}=g_{a}^{*}}
$$

${ }^{9}$ Note from equation (6) that

$$
\lim _{\sigma \rightarrow 1}\left[(\phi A)^{(\sigma-1) / \sigma}+\left((1-\phi) \alpha_{A} L\right)^{(\sigma-1) / \sigma}\right]^{\sigma /(\sigma-1)}=A^{\phi}\left(\alpha_{A} L\right)^{1-\phi},
$$

in which case the model features semi-endogenous growth.

${ }^{10}$ In fact, there is little hope that such an elasticity can ever be recovered econometrically even if all inputs in the R\&D production function could be quantified (i.e., including "knowledge"); cf. the non-identification theorem of Diamond, MacFadden and Rodriguz (1978). Furthermore, even if these empirical complications could be resolved and the evidence points to an elasticity of substitution below one, this does exclude that the elasticity converges to a value above one, which may be sufficient for endogenous growth.

${ }^{11}$ Often, the rate of convergence is formulated in levels, rather than in terms of growth rates. However, in comparing the predictions of the model with the empirical evidence, these two formulations are equivalent. To see this, note that a standard cross-country regression would be

$$
g_{y}^{t} \equiv \ln y_{t}-\ln y_{t-1}=\lambda \ln y_{t-1}+\gamma x^{t},
$$

where the estimate for $\lambda$ can be translated into a rate of convergence, with $x$ a vector of additional controls. Now, take the difference between this equation and the lagged version. Then,

$$
\Delta g_{y} \equiv g_{y}^{t}-g_{y}^{t-1}=\lambda g_{y}^{t-1}+\gamma\left(x^{t}-x^{t-1}\right),
$$

which corresponds to the approach taken here. Accordingly, empirical estimates for $\lambda$ can equally well be capturing the speed of convergence in levels as in growth rates.

(C) The editors of the Scandinavian Journal of Economics 2003. 


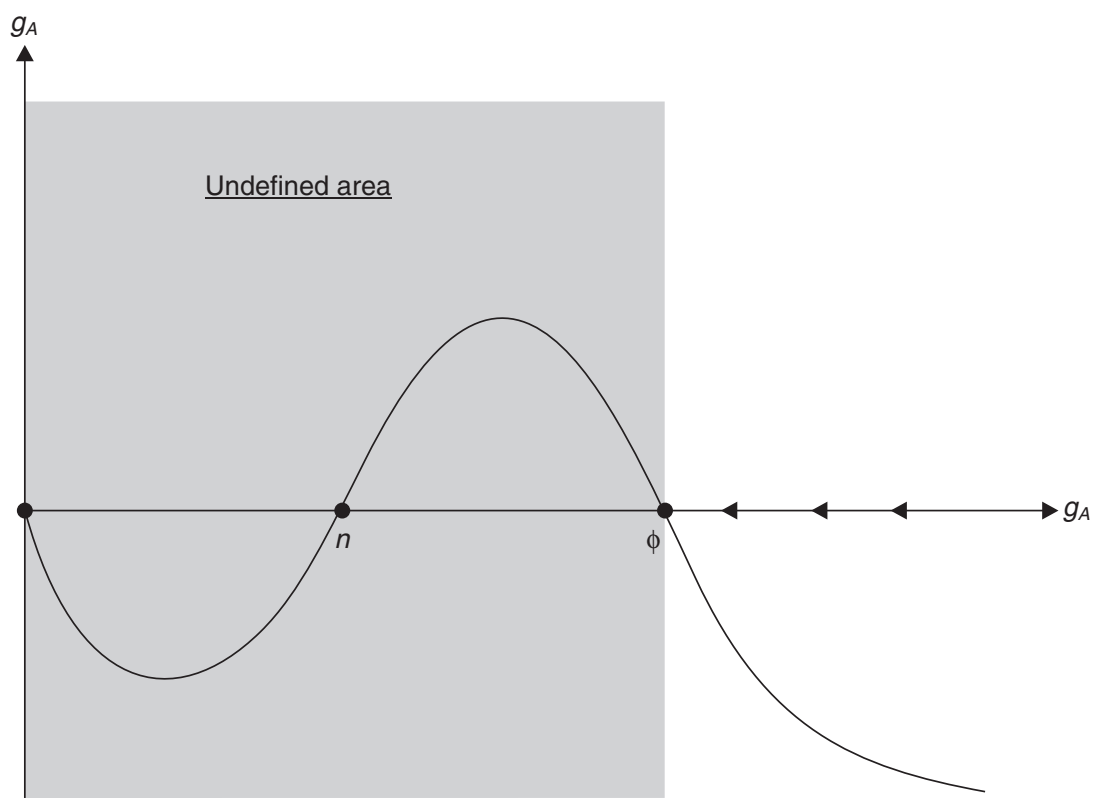

Fig. 2a. Dynamics when $\sigma>1$

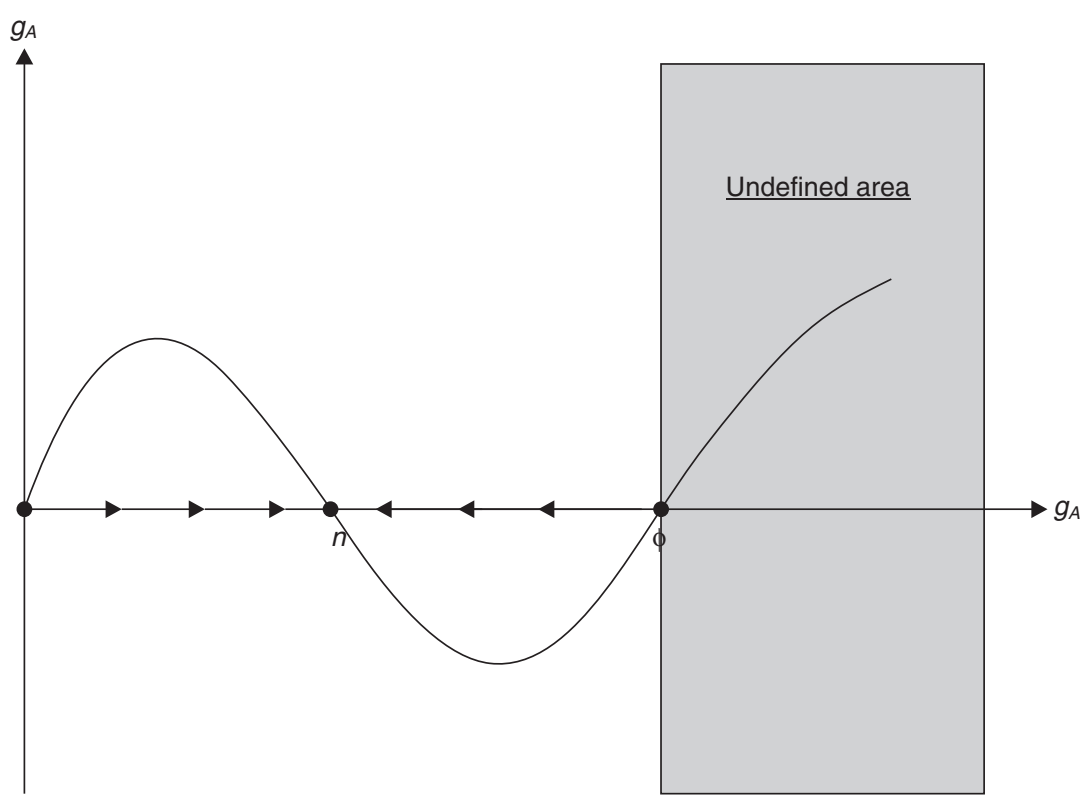

Fig. 2b. Dynamics when $\sigma<1$ 
Hence, the rate of convergence and the condition for local stability are identical expressions. Now, consider again the commonly used functional form $H\left(A, L ; \alpha_{A}\right)=\delta A^{\epsilon}\left(\alpha_{A} L\right)^{\eta}$. In the endogenous growth case, $\epsilon=1$, convergence to the steady-state growth rate is instantaneous. In contrast, the speed of convergence in the semi-endogenous growth model is given by $\eta n$, reflecting the fact that the rate of knowledge growth only gradually adjusts to its long-run rate. However, with this specialized production function for knowledge, the elasticity of substitution is restricted to equal one.

If we instead consider the more general production function (6) then the case of endogenous growth, $\sigma>1$, also displays a gradual adjustment towards the steady-state growth rate. More specifically, the rate of convergence is $[(\sigma-1) / \sigma](\phi-n)$ in the endogenous growth case, $\sigma>1$, compared with $\left[1-(\phi / n)^{(\sigma-1) / \sigma}\right] n$ in the case of semi-endogenous growth, $\sigma<1$. In this example the speed of convergence under endogenous growth lies in the range zero to infinity, while it lies in the range zero to $n$ in the semiendogenous growth case. Without additional information about the parameters, $\phi$ and $\sigma$, matters cannot be pushed much further without becoming very speculative in nature. But this illustration does suggest that, a priori, the speed of convergence in the endogenous growth case is as flexible as that of the semi-endogenous growth model. This impression mirrors the conclusion emerging from recent work on implied rates of convergence within multi-sector general equilibrium endogenous and semi-endogenous growth models. Eicher and Turnovsky (1999b) show that the semi-endogenous growth model allows for speeds of convergence for output per capita in line with empirical findings, i.e., in the vicinity of 3 percent. A corresponding result, however, is derived for a base-line endogenous growth model augmented with capital installation costs in Ortigueria and Santos (1997). In a similar vein, based on a multi-country endogenous growth model, Howitt (2000) demonstrates that the implied rate of convergence toward steady state is strictly below the rate implied by the neoclassical growth model. Hence, both frameworks appear to be capable of quantitatively matching observed patterns of convergence reasonably well, even when the growth engine is Cobb-Douglas. ${ }^{12}$

With the special functional forms considered above, it is clear from equations (3) and (4) that economic incentives and policy can affect steady-state growth in per capita income only in the case of endogenous

\footnotetext{
${ }^{12} \mathrm{~A}$ separate issue raised by Eicher and Turnovsky (1999b) is whether theories of growth are consistent with differential rates of convergence in output and technology, respectively. They show that two-sector semi-endogenous growth models can have this property. While most endogenous growth models constrain output and technology to accumulate in proportion, the model developed by Howitt (2000) is also capable of generating such asymmetries.
}

(C) The editors of the Scandinavian Journal of Economics 2003. 
growth. This feature has been criticized by Jones (1995a) on the grounds that growth in several OECD countries has been fairly constant for decades, while e.g. tax policies have changed quite extensively. Jones (1995b) argues that the semi-endogenous growth model, on the other hand, is capable of matching the facts.

First, note that the condition for semi-endogenous growth, $\tilde{h}\left(L ; \alpha_{A}\right)=0$, implies that the importance of the shift parameter, $\alpha_{A}$, vanishes asymptotically. Or to put it differently, economic incentives, policy and institutions in general do not have any systematic impact in the long run on the marginal product of knowledge in producing new knowledge, and therefore have no effects on long-run income growth. ${ }^{13}$ Although this is consistent with the above facts, the question is whether observing the lack of co-movements between some economic variables is sufficient to exclude once and for all that some economic factors may matter for long-run growth. ${ }^{14}$

Second, in the endogenous growth variant $\alpha_{A}$ influences the long-run growth rate only insofar as $\tilde{h}$ depends on this parameter. If $\tilde{h}$ as well as the elasticities $F_{1} A / Y$ and $F_{2} L / Y$ are independent of $\alpha_{A}$, endogenous growth occurs but policy cannot affect growth permanently. Indeed, this property arises in our CES example where the long-run growth rate equals $\phi$ when $\sigma>1$; in this case, policy can only temporarily affect the growth rate, as in the semi-endogenous growth model.

Third, it may be asked whether observations of changes in different policy variables during a period with fairly constant growth rates imply that these policy variables do not affect growth. It is easy to see that this assertion-at least in principle - is wrong. Suppose, for example, that the knowledge production function exhibits diminishing returns to knowledge, as in the case of curve 2 in Figure 1. Then growth-enhancing policies may simply work to counteract a tendency toward declining growth rates that would otherwise have prevailed. In addition, it can be argued that regressions showing that the growth rate is independent of the tax rate may be suffering from omitted variable bias. It may well be the case that a higher tax rate has a significant negative effect on the growth rate, but that this is roughly offset by a significant positive growth effect of the productive government expenditure that is financed by the higher tax rate, thus resulting in a small overall net effect. In fact, the welfare-maximizing tax rate in a simple Barro (1990)

\footnotetext{
${ }^{13}$ Note, however, that growth may be influenced indirectly by e.g. economic policies if they change the elasticities in the production functions, as first pointed out by Eicher and Turnovsky (1999a). Formally this arises in our model if the elasticities $F_{1} A / Y, F_{2} L / Y, g_{1} A / g$ and $g_{2} L / g$ depend on the $\alpha$ parameters. However, this does not lead to any obvious policy prescriptions since there is no theoretical relationship between the elasticities and the parameters.

${ }^{14}$ In fact, recent empirical studies such as Kocherlakota and Yi (1997) and Kneller, Bleaney and Gemmell (1999) do find that permanent changes in economic policy have permanent effects on income growth rates.
} 
model of productive government expenditure coincides with the growthmaximizing tax rate, so that if the tax rate is close to optimal, there should be little effect on the growth rate-precisely as the observation by Jones (1995b) seems to suggest. ${ }^{15}$

\section{Concluding Remarks}

Is it unrealistic to assume constant returns to knowledge in producing new ideas? While it is tempting to conclude so when focusing on specific functional forms, as is normally done in the endogenous growth literature, we have argued that this conclusion is unwarranted. In fact, such production technologies arise naturally in the long run if one is willing to subscribe to the presumption that new pieces of knowledge always lead to the formation of some new ideas. In addition, we have argued that endogenous growth is consistent with available evidence on rates of convergence and policy effectiveness. Thus, endogenous growth theory should not be regarded as an implausible knife-edge case.

\section{References}

Aghion, P. and Howitt, P. (1992), A Model of Growth through Creative Destruction, Econometrica 60, 323-351.

Barro, R. (1990), Government Spending in a Simple Model of Endogenous Growth, Journal of Political Economy 98, S103-S126.

Dalgaard, C. J. and Kreiner, C. T. (2001), Is Declining Productivity Inevitable?, Journal of Economic Growth 6, 187-203.

Diamond, P., McFadden, D. and Rodriguz, M. (1978), Measurement of the Elasticity of Factor Substitution and Bias of Technical Change, in M. Fuss and D. McFadden (eds.), Production Economics: A Dual Approach to Theory and Applications, Vol. II, North-Holland, Amsterdam.

Eicher, T. S. and Turnovsky, S. J. (1999a), Non-scale Models of Economic Growth, Economic Journal 109, 394-415.

Eicher, T. S. and Turnovsky, S. J. (1999b), Convergence in a Two-sector Nonscale Growth Model, Journal of Economic Growth 4, 413-428.

Frankel, M. (1962), The Production Function in Allocation and Growth: A Synthesis, American Economic Review 52, 995-1022.

Grossman, G. M. and Helpman, E. (1991), Innovation and Growth in the Global Economy, MIT Press, Cambridge, MA.

\footnotetext{
${ }^{15}$ We thank an anonymous referee for suggesting this argument. In this context, it is worth noting that the study by Kocherlakota and Yi (1997) demonstrates a negative effect from taxes on growth only after controlling for the expenditure side.
}

(C) The editors of the Scandinavian Journal of Economics 2003. 
Howitt, P. (1999), Steady Endogenous Growth with Population and R\&D Inputs Growing, Journal of Political Economy 107, 715-730.

Howitt, P. (2000), Endogenous Growth and Cross-country Income Differences, American Economic Review 90, 829-846.

Jones, C. I. (1995a), Time Series Tests of Endogenous Growth Models, Quarterly Journal of Economics 110, 495-525.

Jones, C. I. (1995b), R\&D-based Models of Economic Growth, Journal of Political Economy 103, 759-783.

Jones, C. I. (1999), Growth: With or Without Scale Effects?, American Economic Review, Papers and Proceedings 89, 139-144.

Jones, L. E. and Manuelli, R. E. (1990), A Convex Model of Equilibrium Growth: Theory and Policy Implications, Journal of Political Economy 98, 1008-1038.

Kneller, R., Bleaney, M. and Gemmell, N. (1999), Fiscal Policy and Growth: Evidence from OECD Countries, Journal of Public Economics 74, 171-190.

Kocherlakota, N. R. and Yi, K.-M. (1997), Is there Endogenous Long-run Growth? Evidence from the United States and the United Kingdom, Journal of Money, Credit, and Banking 29, 235-262.

Li, C.-W. (2000), Endogenous vs. Semi-endogenous Growth in a Two-R\&D-sector Model, Economic Journal 110, C109-C122.

Li, C.-W. (2002), Growth and Scale Effects: The Role of Knowledge Spillover, Economics Letters 74, 177-185.

Ortigueria, S. and Santos, M. (1997), On the Speed of Convergence in Endogenous Growth Models, American Economic Review 87, 383-399.

Pitchford, J. D. (1960), Growth and the Elasticity of Factor Substitution, Economic Record 36, 491-504.

Romer, P. (1986), Increasing Returns and Long-run Growth, Journal of Political Economy 94, 1002-1037.

Romer, P. (1990), Endogenous Technical Change, Journal of Political Economy 98, 71-102.

Segerstrom, P. (1998), Endogenous Growth without Scale Effect, American Economic Review 88, 1290-1310.

Solow, R. M. (1994), Perspectives on Growth Theory, Journal of Economic Perspectives 8, $45-54$.

United Nations (1998), World Population Projections to 2150, Department of Economic and Social Affairs, Population Division, New York.

Weitzman, M. L. (1998), Recombinant Growth, Quarterly Journal of Economics 113, 331-360.

Young, A. (1998), Growth without Scale Effects, Journal of Political Economy 106, 41-63.

First version submitted May 2001;

final version received August 2002. 
